\title{
Effet de la nature du foin des Alpes du Nord sur les performances des vaches laitières *
}

\author{
A Hauwuy 1, JB Coulon 2, J Paradis 1 \\ 1 GIS-SUACI Alpes du Nord, 1, rue Métropole, 73000 Chambery ; \\ 2 INRA, Laboratoire d'adaptation des herbivores aux milieux, Theix, \\ 63122 Saint-Genès-Champanelle, France
}

(Reçu le 14 octobre 1992; accepté le 10 mars 1993)

\begin{abstract}
Résumé - Les données concernant 27 vaches laitières, issues d'un essai d'alimentation conduit 2 années consécutives, ont permis de préciser la valeur alimentaire de différents types de foin des Alpes du Nord et de déterminer la réponse des performances des vaches laitières lors du passage d'un type de foin à l'autre. Quatre lots de foin ont été distribués chaque année. Ces foins avaient été récoltés sur des prairies à Ombellifères et Dactyle en touffes ( 5 lots) ou à géranium et Graminées en tapis régulier ( 3 lots). Les valeurs d'encombrement calculées a posteriori ont varié de 0,96 à 1,21 UEL $\mathrm{kg}$ MS. Ces valeurs sont inférieures aux valeurs affectées aux foins de plaine ou de montagne de même valeur nutritive. Pour un type de foin donné, la valeur nutritive des foins testés n'explique pas entièrement la variabilité observée au niveau de leur ingestibilité. Les variations immédiates des performances des vaches laitières lors du passage d'un lot de foin à l'autre ont surtout été nettes sur la production laitière et le taux protéique. Après 1 à 2 semaines d'adaptation, les écarts de performances d'un type de foin à l'autre ont été faibles. Pour le taux protéique, elles ont été significativement liées aux variations correspondantes des apports énergétiques. Cependant, certains foins semblent avoir un effet propre sur le lait ou les taux de matières utiles. Ces résultats demandent à être précisés sur d'autres types de foin et d'autres stades de récolte.
\end{abstract}

vache laitière / foin / ingestibilité / composition du lait

Summary - Influence of hay type on dairy cows' performance. Data from 27 dairy cows (Abundance breed) in a 2-yr experiment were used to examine the fill value of different hays from native mountain grassland (northern alps) and the modifications in the cows' performance when the type of hay was changed. Cows were fed ad libitum with 8 different types of hay (table l). The amount of concentrate was adjusted to meet the nutritive requirements and was not modified when the type of hay was changed. Fill values of the different types of hay calculated from cows' performance varied between 0.96 and 1.21 UEL/kg DM. These values were lower than those of other natural grassland hays (lowland or upland) with the same energy value (fig 1). Immediate variations in milk production $(-1.8$ to $+1.0 \mathrm{~kg} / \mathrm{d})$ and protein content $(-1.9$ to $+1.6 \mathrm{~g} / \mathrm{kg})$ when the type of hay was changed were

* Étude réalisée dans le cadre du programme de Recherche-Développement des Alpes du Nord (SUACI, 1, rue Métropole 73000 Chambéry) 
higher than fat content variations $(-1.0$ to $+1.4 \mathrm{~g} / \mathrm{kg}$ ) (fig 2). After 1 or 2 wk adaptation, these variations in performance from one type of hay to another were low. Protein content variations were significantly related to corresponding energy supply variations $(\mathrm{R}=0.80, \mathrm{P}<0.05)$ (fig 3 ). Nethertheless, some types of hay seem to have a specific effect on milk production or milk composition. These results have to be confirmed on a larger range of hay types.

dairy cow / hay / ingestibility / milk composition

\section{INTRODUCTION}

Le foin est un aliment important en élevage bovin laitier dans les zones herbagères. Lorsque le lait y est transformé en fromage d'appellation, c'est parfois la seule ration de base autorisée. Dans ces régions, la nature de ces foins, essentiellement récoltés sur prairies naturelles, est très variable ; elle dépend, d'une part, des conditions pédoclimatiques du milieu (fraîcheur et réserve calcique du sol) et, d'autre part, des pratiques agricoles (fertilisation organique et/ou minérale et stade de récolte). Dans les Alpes du Nord, les prairies de fauche ont fait l'objet d'une classification et d'une description détaillées, fondées sur l'importance et la nature des dicotylédones et la morphologie des Graminées présentes (Fleury et al, 1986). Par ailleurs, dans le cadre de la transformation du lait en fromage d'AOC, où les caractéristiques originelles du lait ne peuvent en général être modifiées, il est important de préciser si, à côté des effets liés aux niveaux des apports nutritifs, la nature des fourrages n'a pas un effet propre sur les performances des vaches laitières et sur la composition chimique de leur lait en particulier. Un certain nombre de travaux ont, en effet, mis en évidence des écarts de production et/ou de composition du lait entre différentes natures de flore au pâturage (Demarquilly, 1963 ; Thomson et al, 1985 ; Haltel et Bornard 1989) ou entre différents foins ou ensi- lages (Horner et al, 1985 ; Thomas et al, 1985). Ces différences semblent cependant essentiellement dues à des niveaux d'apports énergétiques variables.

L'objectif de cette étude, réalisée dans le cadre d'un essai d'alimentation conduit dans une exploitation privée des Alpes du Nord (Hauwuy et al, 1992), a été de préciser : 1) les caractéristiques alimentaires de foins de différents types, 2) la réponse des performances des vaches laitières lors du passage d'un type de foin à l'autre.

\section{MATÉRIELS ET MÉTHODES}

\section{Conditions générales}

L'essai sur l'alimentation hivernale des vaches laitières, dont est issue la présente étude, a été réalisé au cours de 2 hivers consécutifs. Ses caractéristiques détaillées ont été décrites par ailleurs (Hauwuy et al, 1992). Au cours des 2 périodes hivernales, les 48 vaches laitières disponibles (dont 16 primipares) recevaient une ration de base composée de foin de premier cycle de végétation offert à volonté (refus > 10\%) en 2 repas par jour et d'une quantité fixe de regain (2 à $4 \mathrm{~kg} / \mathrm{j}$, selon le type de foin associé). Cette ration était complémentée de manière à réaliser, à partir de la deuxième semaine de lactation des animaux, 2 niveaux de couverture des besoins énergétiques (haut : couverture des besoins [lot $\mathrm{H}$; ; bas : $2 \mathrm{~kg} / \mathrm{j}$ de concentré en moins par rapport au lot $H$ [lot $B]$ ). Le rationnement des animaux en aliment concentré était individualisé et prédéterminé pour toute la période hivernale. La composition de l'aliment concentré (mélange de 
céréales aplaties et de tourteau de soja) était ajustée, de manière à ce que les apports azotés soient identiques dans les 2 lots et couvrent les besoins des animaux dès le vêlage.

\section{Caractéristiques des animaux et des fourrages retenus}

Parmi les 48 vaches disponibles, 27 ont été utilisées pour cette étude (respectivement 14 et 13 des lots $\mathrm{H}$ et $\mathrm{B}$ ). Au début de la période d'étude retenue (début février à début mai), elles étaient au moins en huitième semaine de lactation. Elles n'ont présenté aucun trouble sanitaire au cours de cette période. La proportion de primipares a été importante (13/27) du fait de leur date de vêlage plus précoce.

Chaque hiver, 4 lots de foins différents ont été étudiés (foins $11,12,13$ et 14 au cours du premier hiver ; $21,22,23$ et 24 au cours du second hiver) au cours de 13 semaines de distribution consécutives. Le foin de chacun des lots a été distribué au cours de périodes de mesures de 2 à 7 semaines. Ces foins avaient été récoltés sur des prairies naturelles, dont la végétation a été caractérisée selon la typologie des prairies de fauche des Alpes du Nord humides (Fleury et al, 1986). Les prairies étaient soit des prairies à Ombellifères et Dactyle en touffes (type M1B1, 5 lots de foin), soit des prairies à géranium et Graminées en tapis régulier (type M3, 3 lots de foin). Les différents lots de foin, récoltés en vrac et séchés en grange, avaient été identifiés et séparés lors du stockage. Le regain avait été récolté sur l'ensemble des parcelles de fauche de l'exploitation. Les apports de regain ont été de $4 \mathrm{~kg} / \mathrm{j}$, sauf au cours d'une période de la seconde année (période 23 ), où ils ont été réduits à $2 \mathrm{~kg} / \mathrm{j}$. Les 10 premiers jours de cette même période, les apports de tourteau de soja ont aussi été réduits de $0,5 \mathrm{~kg} / \mathrm{j}$. Le passage d'un type de foin à l'autre a été effectué sans transition, du jour au lendemain.

\section{Mesures}

Les quantités de lait produites ont été pesées individuellement, matin et soir, $3 \mathrm{j}$ par semaine. Les taux butyreux et protéique ont été déterminés une fois par semaine sur un échantillon moyen de 2 traites consécutives. Les vaches ont été pesées toutes les 2 semaines. Les quantités d'aliments offertes et refusées ont été pesées individuellement pour chaque type d'aliment 3 j consécutifs par quinzaine. Pour chaque lot de foin, ainsi que sur les refus correspondants et le regain, une analyse de la composition chimique et une mesure de la digestibilité de la matière organique (méthode enzymatique de Aufrère et Michalet-Doreau, 1983) ont été réalisées à la récolte et lors de la distribution (tableau I). On en a déduit leur valeur nutritive, à partir des équations d'Andrieu et Demarquilly (1987).

\section{Analyse des données}

Les apports nutritifs disponibles ont été calculés pour chaque période en tenant compte de la valeur nutritive des refus. Les apports énergétiques ont été corrigés pour tenir compte des interactions métaboliques et digestives (Vermorel et al, 1987).

Pour l'estimation de la valeur d'encombrement des foins, la capacité d'ingestion thérique des animaux, en fonction de leur production laitière et de leur poids vif moyen au cours de chaque période de distribution, a été calculée ( $\mathrm{Fa}$ verdin et al, 1987). Compte tenu du faible effet du numéro de lactation sur les quantités ingérées, une fois pris en compte l'effet du poids et de la production laitière (Hauwuy et al, 1992), le même modèle de prévision a été appliqué aux primipares et aux multipares. Cette simplification a cependant pu conduire à une légère sousestimation de l'ingestibilité des foins étudiés. La valeur d'encombrement (UEL $/ \mathrm{kg}$ MS) du fourrage (foin + regain) a été calculée à l'aide du logiciel INRAtion (Coulon et Faverdin, 1993). Elle correspond aux quantités de fourrage réellement ingérées. La valeur d'encombrement de chacun des foins a été obtenue en fixant une valeur de 0,95 UEL/kg MS au regain. Compte tenu de la part de regain dans la ration, la variation de la valeur UEL du regain de 0,1 n'entraîne qu'une variation de la valeur UEL du foin d'environ 0,03 .

L'effet des changements de lot de foin a été étudié, d'une part, au cours de la première semaine de distribution et, d'autre part, après une période d'adaptation d'au moins 1 semaine. Dans le premier cas, les variations individuelles 
Tableau I. Caractéristiques, composition chimique et valeur nutritive des fourrages à la distribution.

Année 1

Année 2

Foin 11 Foin 12 Foin 13 Foin 14 Regain Foin 21 Foin 22 Foin 23 Foin 24 Regain

\begin{tabular}{|c|c|c|c|c|c|c|c|c|c|c|}
\hline $\begin{array}{l}\text { Nature * } \\
\text { Stade de fauche }\end{array}$ & $\begin{array}{l}\text { M1B1 } \\
\text { P Ep }\end{array}$ & $\begin{array}{l}\text { M3 } \\
\text { Fruc }\end{array}$ & $\begin{array}{l}\text { M1B1 } \\
\text { P Ep }\end{array}$ & $\begin{array}{l}\text { M3 } \\
\text { Fruc }\end{array}$ & & $\begin{array}{l}\text { M1B1 } \\
\text { D Fruc }\end{array}$ & $\begin{array}{l}\text { M1B1 } \\
\text { Mi FI }\end{array}$ & $\begin{array}{l}\text { M1B1 } \\
\text { P Ep F }\end{array}$ & $\begin{array}{l}\text { M3 } \\
\text { ruc }\end{array}$ & \\
\hline Distribution (sem) & 2 & 2 & 7 & 2 & 13 & 3 & 4 & 3 & 3 & 13 \\
\hline \multicolumn{11}{|c|}{ Composition chimique (\% MS) } \\
\hline MO & 91,0 & 92,6 & 90,5 & 91,4 & 87,9 & 91,5 & 93,8 & 91,0 & 93,4 & 88,3 \\
\hline MAT & 9,3 & 6,9 & 8,8 & 7,4 & 13,5 & 7,1 & 8,6 & 8,8 & 7,1 & 14,2 \\
\hline $\mathrm{CB}$ & 29,1 & 34,2 & 30,7 & 32,7 & 23,6 & 33,8 & 38,0 & 35,1 & 40,6 & 25,7 \\
\hline $\mathrm{dMO} \mathrm{O}^{\star \star \star}$ & 64 & 56 & 60 & 56 & 68 & 56 & 51 & 59 & 55 & 70 \\
\hline \multicolumn{11}{|c|}{ Valeur nutritive (kg MS) ${ }^{* * * *}$} \\
\hline UFL & 0,75 & 0,64 & 0,69 & 0,63 & 0,79 & 0,63 & 0,58 & 0,67 & 0,62 & 0,82 \\
\hline PDIN (g) & 58 & 43 & 55 & 46 & 84 & 44 & 53 & 55 & 44 & 88 \\
\hline PDIE (g) & 72 & 63 & 70 & 64 & 85 & 63 & 65 & 67 & 60 & 85 \\
\hline
\end{tabular}

$\because$ Typologie des prairies de fauche des Alpes du Nord humides (Fleury et al, 1986): M1B1: foins a anthrisque; M3: foins à géranium. **: Stades phénologiques du Dactyle: P Ep: pleine épiaison; D Fruc: début fructification; Mi FI: Mifloraison; Fruc: fructification; "**: Méthode enzymatique (Pepsine cellulase O1N) (Aufrère et Michalet-Doreau, 1983); "**: D'après Andrieu et Demarquilly, 1987.

de production et de composition chimique du lait d'une semaine à l'autre ont été analysées, par comparaison de moyenne (test de Dunnett, logiciel STATITCF) afin de mettre en évidence si les variations correspondant aux changements de foin étaient significativement différentes des variations aléatoires. Dans le second cas, on a calculé les écarts hebdomadaires entre les performances moyennes observées et les valeurs obtenues par régression sur l'ensemble de la période, hormis la première semaine de distribution de chaque lot de foin. Le même calcul a été réalisé pour les apports nutritifs (UFL et PDI). Les écarts moyens de performances au cours des 2 dernières semaines de chaque période (ou de la dernière semaine dans les cas des périodes n'excédant pas 2 semaines) ont alors été comparés avec les écarts d'apports nutritifs correspondants. Le foin 14, pour lequel il n'y a pas eu de mesures des quantités ingérées au-delà de la première semaine de distribution n'a pas été retenu dans cette analyse.

Les variations des performances des animaux lors des changements de foin n'ayant pas été différentes selon le niveau d'apport énergétique de l'aliment concentré qu'ils recevaient, l'évolution de ces performances au cours des 13 semaines expérimentales a été illustrée par la moyenne de l'ensemble des animaux retenus.

\section{RÉSULTATS ET DISCUSSION}

\section{Composition chimique et valeur nutritive des foins}

Les foins à anthrisque ont été récoltés la première année, au stade début épiaison du Dactyle, alors que la deuxième année leur stade de fauche a été variable (tableau I). Quelle que soit l'année, les foins à géranium ont été récoltés plus tardivement (stade fructification du dactyle). Les différences de stade de récolte, mais aussi de composition de la végétation inter- ou 
même intratype de prairie, ont entraîné des différences de composition chimique et de valeur nutritive importantes entre les lots de foin. Les teneurs en matière azotée totale et en cellulose brute des foins de premier cycle ont varié respectivement de 7,4 à $9,3 \%$ MS et de 19,1 à $40,6 \%$ MS. La valeur énergétique de ces foins a varié de 0,58 à 0,75 UFL/kg MS, la valeur PDIN de 43 à $58 \mathrm{~g} / \mathrm{kg}$ MS et la valeur PDIE de 63 à $72 \mathrm{~g} / \mathrm{kg}$ MS. En moyenne, les foins de la deuxième année ont été plus pauvres en énergie et en azote, du fait de leur stade de fauche plus tardif.

\section{Valeur d'encombrement des foins}

Les valeurs d'encombrement calculées pour les 8 lots de foin utilisés ont varié de 0,96 à 1,21 UEL/kg MS (tableau II). Conformément aux équations de prévisions établies pour les foins de montagne (Dulphy et al, 1987), elles ont été d'autant plus faibles que la digestibilité de la matière organique (et de ce fait la valeur énergétique correspondante) a été plus élevée

Tableau II. Valeurs d'encombrement des différents lots de foin (calculées en affectant une valeur de 0,95 UEL/kg MS au regain).

$\begin{array}{cccc}\text { Foin } & \begin{array}{c}\text { Type } \\ \text { (1) }\end{array} & \begin{array}{c}\text { Valeur } \\ \text { energétique } \\ \text { (UFL/kg MS) }\end{array} & \begin{array}{c}\text { Valeur } \\ \text { d'encombrement } \\ \text { (UEL/kgMS) }\end{array}\end{array}$

\begin{tabular}{|c|c|c|c|}
\hline \multicolumn{4}{|c|}{ Année 1} \\
\hline 11 & M1B1 & 0,75 & 0,96 \\
\hline 12 & M3 & 0,64 & 1,09 \\
\hline 13 & M1B1 & 0,69 & 1,03 \\
\hline 14 & M3 & 0,63 & 1,00 \\
\hline \multicolumn{4}{|c|}{ Année 2} \\
\hline 21 & M1B1 & 0,63 & 1,03 \\
\hline 22 & M1B1 & 0,58 & 1,21 \\
\hline 23 & M1B1 & 0,67 & 1,12 \\
\hline 24 & M3 & 0,62 & 1,03 \\
\hline
\end{tabular}

(fig 1). Pour une même valeur énergétique, ces foins se caractérisent par une ingestibilité légèrement supérieure à celle des foins de prairie naturelle de demimontagne ( $+2 \%$ environ) et de plaine $(+8 \%$ environ), récoltés dans les mêmes conditions (foins ventilés) (INRA, 1988). Ces écarts semblent d'autant plus importants que la valeur énergétique des foins est plus élevée (fig 1). Ils atteignent, d'autre part, près de $20 \%$ quand on compare les valeurs de cette étude à celles provisoirement affectées aux foins des Alpes du Nord riches en dicotylédones, obtenues il est vrai dans des conditions différentes (animaux forts producteurs et non habitués à ce type de fourrage, foins récoltés en vrac et mis en bottes pour le transport).

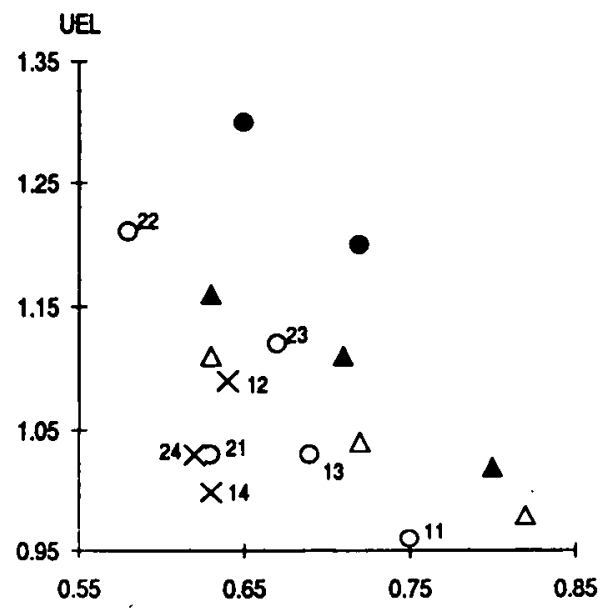

Fig 1. Relation entre les valeurs UEL et UFL des foins et comparaison avec celles de prairies permanentes récoltées dans des conditions comparables (INRA, 1988). $)$ : foins à anthrisque ( $n^{\circ} 11,13,21,22$ et 23 ); $X$ : foins à géranium ( $n^{\circ} 12,14$ et 24$) ; \Delta$ : foins de demimontagne (Auvergne); $\mathbf{\Delta}$ : foins de plaine (Normandie); : foins de montagne (Alpes du Nord) à base de dicotylédones. 
Ces ingestibilités élevées confirment les résultats observés sur moutons pour des foins comparables (Dulphy, 1988, non publié). Elles sont certainement dues en partie au fait que le foin était réellement distribué à volonté, permettant aux animaux de trier, pratique d'autant plus importante que le foin présente une plus forte variabilité dans ses différents composants. Selon les foins, la valeur énergétique des refus a ainsi été inférieure de 0 à $23 \%$ à celle de l'offert ( $12 \%$ en moyenne), et cet écart est d'autant plus important que l'ingestibilité du foin correspondant est plus élevée $(R=$ $0,77, P<0,01)$.

Ces résultats montrent, par ailleurs, qu'il peut persister une variabilité importante de l'ingestibilité, indépendamment de la valeur énergétique (cas des foins 12 et 14 d'une part, et 21 et 23 d'autre part) (fig 1). Pour les foins à anthrisque (type M1B1), la proportion de dicotylédones, qui peut varier de façon importante d'un lot de foin à l'autre (foin $21: 40 \%$, foin $23: 60 \%$ ), peut expliquer une part de cette variabilité. La faible plage de variation de la valeur nutritive des foins à géranium (type M3) n'a pas permis de préciser si le type de foin pouvait expliquer en partie cette variabilité.

\section{Effets immédiats des changements de foin}

Les changements de lots de foin distribués ont entraîné des modifications sensibles des performances des animaux concernant essentiellement la production laitière $(-1,8 \mathrm{~kg} / \mathrm{j}$ par exemple lors du passage du foin 13 au foin 14) et le taux protéique $(-1,9 \mathrm{~g} / \mathrm{kg}$ lors du passage du foin 21 au foin 22) (fig 2) qui ont été significatives $(P<0,05)$ dans 4 des 6 changements observés (tableau III). Pour le taux butyreux, les variations lors des changements de foin n'ont jamais été significativement différentes de celles observées au cours des autres semaines, vraisemblablement en raison de la grande variabilité de ce taux. Les variations des performances ont été immédiates : elles ont été perceptibles dès la première semaine de distribution.

Tableau III. Variations immédiates des performances des animaux lors des changements de lot de foin.

\begin{tabular}{lccc}
\hline & $\begin{array}{l}\text { Lait } \\
(\mathrm{kg} / \mathrm{j})\end{array}$ & $\begin{array}{l}\text { Taux butyreux } \\
(\mathrm{g} / \mathrm{kg})\end{array}$ & $\begin{array}{c}\text { Taux protéique } \\
(\mathrm{g} / \mathrm{kg})\end{array}$ \\
\hline Année 1 & & & 0,2 \\
Témoin (1) & 0,1 & 0,3 & $-0,9$ \\
Changement 1 & $-0,1$ & $-1,1$ & $1,6^{*}$ \\
Changement 2 & $-0,5$ & 1,4 & $-1,7^{*}$ \\
Changement 3 & $-1,8^{*}$ & 0,3 & \\
Année 2 & & & $-0,2$ \\
Témoin (1) & $-0,2$ & 0,0 & $-1,9^{*}$ \\
Changement 1 & $-1,4^{*}$ & $-1,1$ & $-0,3$ \\
Changement 2 & $-1,4^{*}$ & $-0,1$ & $1,3^{*}$ \\
Changement 3 & $1,0^{*}$ & $-0,1$ & \\
\hline
\end{tabular}

Les valeurs suivies d'un astérisque sont significativement différentes $(P<0,05)$ du témoin. (1) Moyenne des variations hebdomadaires en l'absence de changement de lot de foin. 


\section{Performances des animaux selon les lots de foin}

Les variations des apports nutritifs d'un lot de foin à l'autre reflètent celles de la valeur nutritive des fourrages, associées (foin 13, 22 et 24 ) ou non (foin 12 et 23 ) à des mo- difications de l'ingestion de ces fourrages (fig 2).

Après 1 à 2 semaines d'adaptation, les variations de performances liees à la nature du foin ont été faibles, en particulier pour le lait et le taux butyreux ; pour 6 des 7 lots de foins étudiés, ces variations ont
Année 1
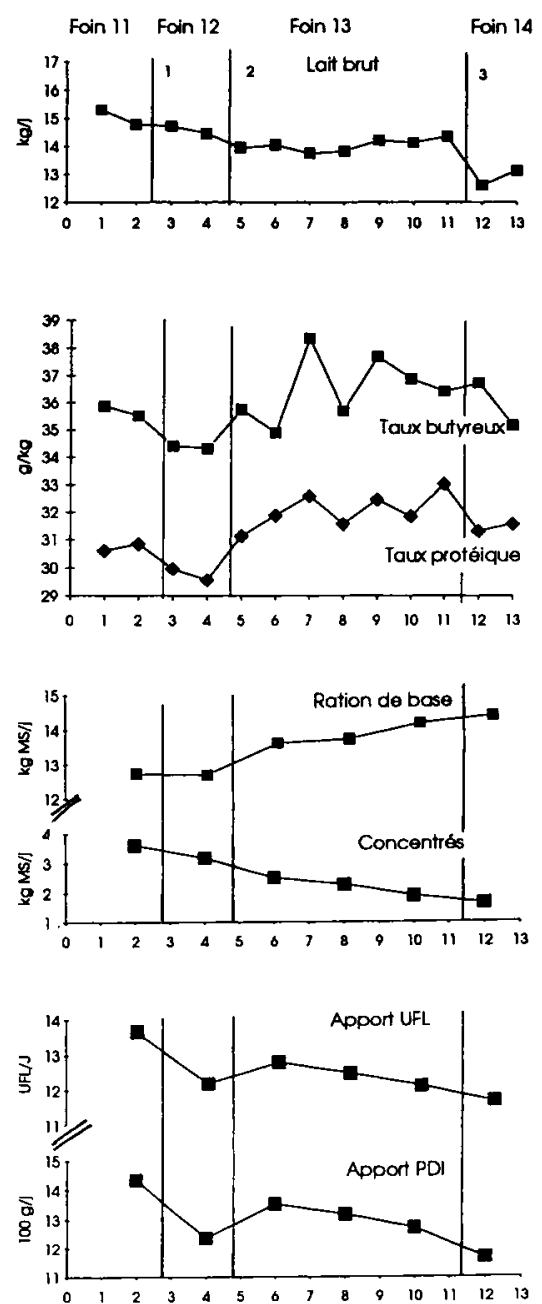

Semaines
Année 2
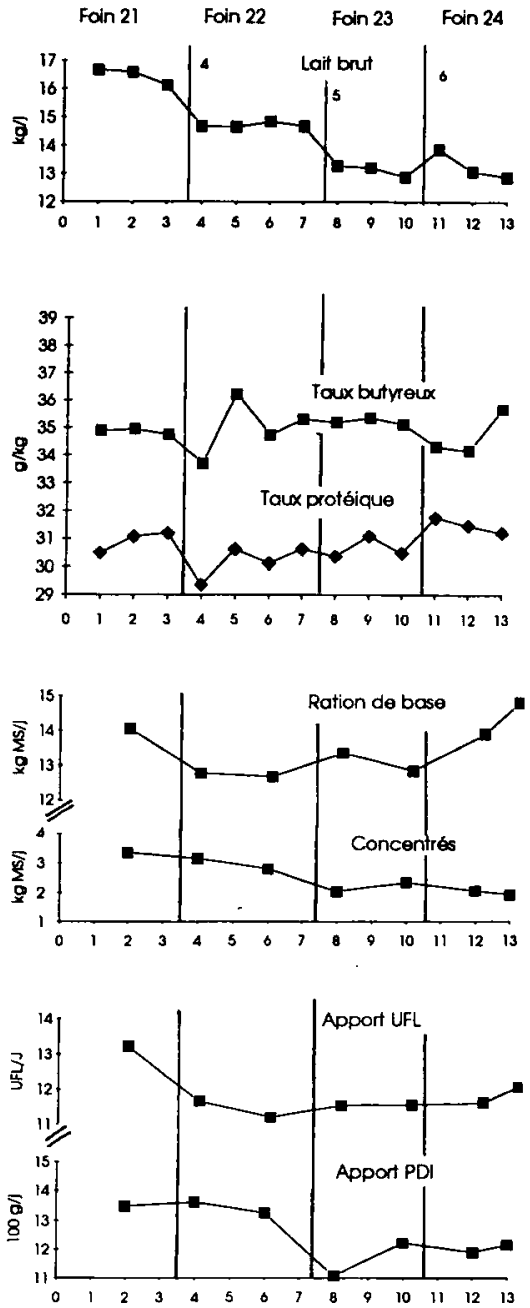

Semaines

Fig 2. Évolution des performances des animaux au cours des 2 années. 
été comprises entre 0,0 et $0,4 \mathrm{~kg} / \mathrm{j}$ pour le lait, $-0,3$ et $+0,3 \mathrm{~g} / \mathrm{kg}$ pour le taux butyreux et $-0,5$ et $+0,4 \mathrm{~g} / \mathrm{kg}$ pour le taux protéique (fig 3). Cette faible variabilité, associée au fait que les animaux étaient en bilans nutritifs largement positifs durant les périodes de mesures (Hauwuy et al, 1992), expliquent que les variations de production laitière et de taux butyreux ne soient pas liées à celles des apports nutritifs corres-
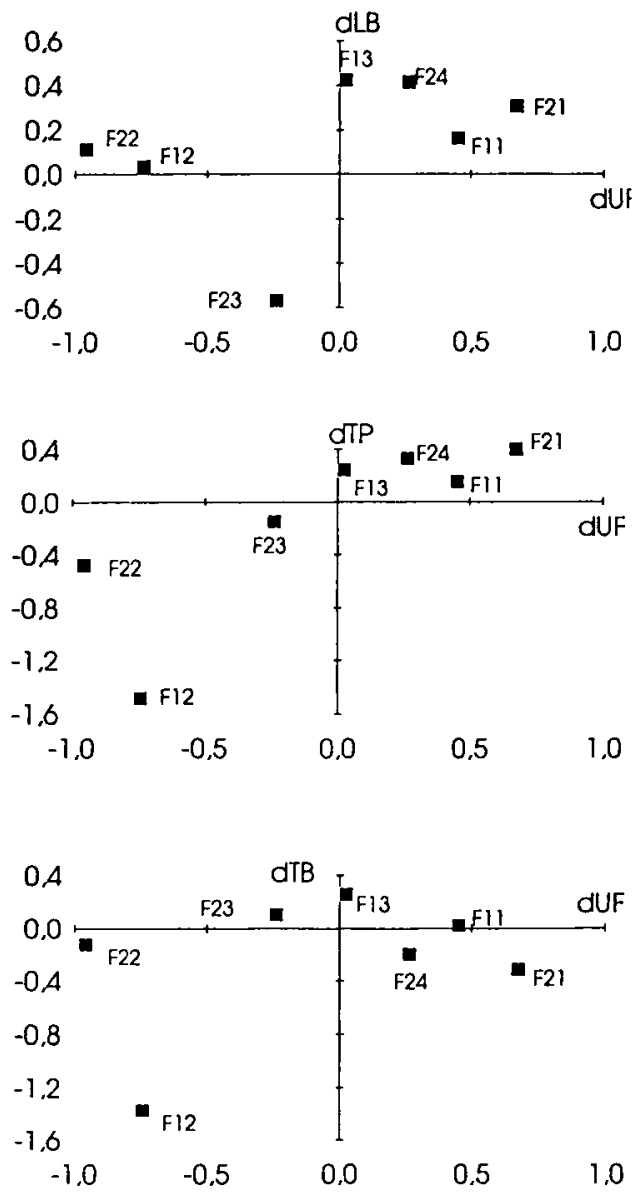

Fig 3. Relations entre les variations de la production laitière (dLB, $\mathrm{kg} / \mathrm{j}$ ), du taux protéique (dTP, $g / \mathrm{kg}$ ) et du taux butyreux (dTB, $g / \mathrm{kg}$ ), et celles des apports énergétiques (dUF, UFLi) selon les différents lots de foin distribués. pondant. Les variations de taux protéique ont, en revanche, été liées significativement à celles des apports énergétiques $(\mathrm{R}=0,80, P<0,01$ ) (fig 3 ), comme cela est couramment observé (Coulon et Rémond, 1991).

La seule diminution sensible de la production laitière a été observée lors de la distribution du foin 23 , alors que les apports nutritifs variaient peu, et que la composition du lait restait inchangée. À l'inverse, la distribution du foin 12 , qui a conduit à une nette diminution des apports énergétiques et azotés, s'est accompagnée d'une diminution importante des taux butyreux et protéique, mais non de la production laitière (fig 3 ).

\section{CONCLUSION}

En définitive, cette étude a mis en évidence l'ingestibilité élevée de certains foins caractéristiques des zones de montagne. Elle a, d'autre part, montré que le passage d'un lot de foin à un autre pouvait entraîner des modifications sensibles, mais la plupart du temps de courte durée, des performances de production des vaches laitières. Elle suggère que certains foins pourraient avoir un effet propre sur la production ou la composition chimique du lait. Elle doit cependant être précisée et cornplétée par des observations sur une gamme plus large de foins, intégrant en particulier des foins de types très différents (à base de Graminées) et récoltés à des stades variables.

\section{REMERCIEMENTS}

Nous tenons à remercier $M$ Folliet et Mme Girard-Derpraulet, qui ont mis leur exploitation à notre disposition et ont supporté patiemment les contraintes de l'expérimentation. 


\section{RÉFÉRENCES}

Andrieu J,Demarquilly C (1987) Valeur nutritive des fourrages : tables et prévision. Bull Tech CRZV Theix, INRA 70, 61-74

Aufrère J, Michalet-Doreau B (1983) in vivo digestibility and prediction of digestibility of some by-products. In: Feeding value of byproducts and their use by beef cattle. EEC seminar, Melle Gontrode, Belgique, 2534

Coulon JB, Faverdin P (1993) INRAtion. Programme de rationnement pour les ruminants. 1. Vaches laitières (J Agabriel, P Champciaux, C Espinasse, eds). CNERTA, Dijon

Coulon JB, Rémond B (1991) Réponse de la production et de la composition du lait de vache aux variations d'apports nutritifs. INRA Prod Anim 4, 49-56

Demarquilly C (1963) Influence de la nature du pâturage sur la production laitière et la composition du lait. Ann Zootech 12, 69-104

Dulphy JP, Faverdin P, Micol D, Bocquier F (1987) Révision du système des Unités d'Encombrement (UE). Bull Tech CRZV Theix INRA 70, 35-48

Faverdin P, Hoden A, Coulon JB (1987) Recommandations alimentaires pour les vaches laitières. Bull Tech CRZV Theix INRA 70, 133152
Fleury P, Jeannin B, Dorioz JM (1986) Typologie des prairies de fauche de montagne des Alpes du Nord humides. GIS Alpes du Nord, 1 , rue Métropole, 73000 Chambéry

Haltel L, Bornard A (1989) Production laitière permise par le pâturage de pelouses de haute montagne (Alpes du Nord françaises). 6 réunion du sous-réseau $F A O$ des herbages de montagne, Cracovie, Pologne

Hauwuy A, Paradis J, Coulon JB (1992) Complémentation énergétique des rations à base de foin pour les vaches laitières. INRA Prod Anim 5, 339-346

Horner JL, Bush LJ, Adams GD (1985) Comparative nutritional value of eastern gamagrass and alfalfa hay for dairy cows. J Dairy Sci 68 , 2615-2620

INRA (1988) Alimentation des bovins, ovins et caprins. INRA Publications, Paris

Thomas C, Aston K, Daley SR (1985) Milk production from silage. A comparison of red clover with grass silage. Anim Prod 41, 23-31

Thomson DJ, Beever DE, Haines MJ, Cammell $S B$, Evans RT, Dhanoa MS, Austin AR (1985) Yield and composition of milk from friesian cows grazing either perennial ryegrass or white clover in early lactation. J Dairy Res 52, 17-31

Vermorel M, Coulon JB, Journet M (1987) Révision du système des unités fourragères (UF). Bull Tech CRZV Theix INRA 70, 9-18 